\title{
COVID-19-Schutzimpfung für das Krankenhauspersonal
}

\author{
Eine Begleitbefragung der ersten Impfphase am Klinikum Klagenfurt am Wörthersee. \\ Christine Leber-Anderwald
}

Mit der Freigabe der Impfstoffe durch die Europäische Arzneimittelagentur besteht die Chance, die aktuelle Pandemie zu bewältigen. Voraussetzung dafür ist eine hohe Durchimpfungsrate. Es gibt generell keine Impfverpflichtung, aber eine Impfempfehlung und hier haben vor allem die Mitarbeiterinnen und Mitarbeiter im Gesundheitsbereich eine besondere Verantwortung. ${ }^{1}$

In Anlehnung an die Impfstrategie für Österreich seitens des Bundesministeriums für Soziales, Gesundheit, Pflege und Konsumentenschutz (BMSGPK) und die Empfehlungen des Nationalen Impfgremiums zur Priorisierung in Bezug auf die geplanten COVID-19-Impfungen in den fünf KABEG-Häusern erfolgte im Dezember 2020 eine Erhebung der Impfmotivation in den Kategorie-I-Bereichen (Personal in medizinischen Einrichtungen mit besonders hohem Expositionsrisiko) des Klinikums Klagenfurt am Wörthersee (KKW). ${ }^{2}$

Das Klinikum Klagenfurt bietet als Schwerpunktkrankenhaus das Leistungsspektrum einer Universitätsklinik mit Ausnahme der Transplantationschirurgie. Es ist Lehrkrankenhaus der Medizinischen Universitäten Graz, Wien und Innsbruck und ist in vielen Bereichen zertifiziert (z.B. ISO 9001, EMAS etc.). Das drittgrößte Krankenhaus Österreichs verfügt über 1344 Betten und des Weiteren über 126 Betten für chronisch Kranke. Insgesamt sind im KKW mehr als 4000 Menschen beschäftigt.

Eine Priorisierung aus medizinisch-fachlicher Sicht wird empfohlen, um jene Personen frühestmöglich mit Impfstoffen gegen COVID-19 zu schützen, welche ein besonders hohes beruflich bedingtes Ansteckungsrisiko bei gleichzeitiger Systemrelevanz haben. Im KKW zählen folgende Bereiche dazu: die zen- trale Notaufnahme, Bereiche der medizinischen Betreuung von COVID-19-Patientinnen und -Patienten, testendes Personal, Beschäftigte aus Bereichen, in denen infektionsrelevante Tätigkeiten durchgeführt werden, z.B. Abstrichnahme, Personal in Labors mit Verarbeitung von COVID-19-Proben und die Intensivstationen. ${ }^{3}$

Im Rahmen dieser Erhebung kam es zu einer Impfbereitschaft von 40 Prozent der Mitarbeiter im KKW und 80 Prozent (6321 Mitarbeiter) der Belegschaft in den gesamten fünf Häusern der KABEG.

In der zweiten Kalenderwoche 2021 begann seitens des Arbeitgebers eine Informations- und Aufklärungskampagne rund um die COVID-19-Schutzimpfung (Experteninterviews via soziale und elektronische Medien, Informationsbroschüren, Informationshotline...). ${ }^{4}$

\section{Phasen der Impfung}

In der Phase 0 wurden die Mitarbeiter der Abteilungen für chronisch Kranke geimpft (Lieferung der Impfdosen analog den Bewohnern und Mitarbeitern in Pflegeheimen). Phase 1 startete in den KABEG-Spitälern am 19.1. 2021 mit der Impfung der Mitarbeiter der Kategorie I.

Es wurden im Klinikum Klagenfurt 819 Mitarbeiterinnen und Mitarbeiter geimpft (69 Prozent der weiblichen Mitarbeiterinnen und 31 Prozent der männlichen Mitarbeiter) und die Impfaktion wurde mit einer Fragebogenerhebung zur persönlichen Impfmotivation der Mitarbeiter begleitet. Dazu wurde die Methode der Querschnittsstudie gewählt. In der empirischen Forschung spricht man von einem Querschnittsdesign, wenn eine empiri-

\section{ABB. 1: INFORMATIONSQ̣UELLEN FÜR IHRE URTEILSBILDUNG}

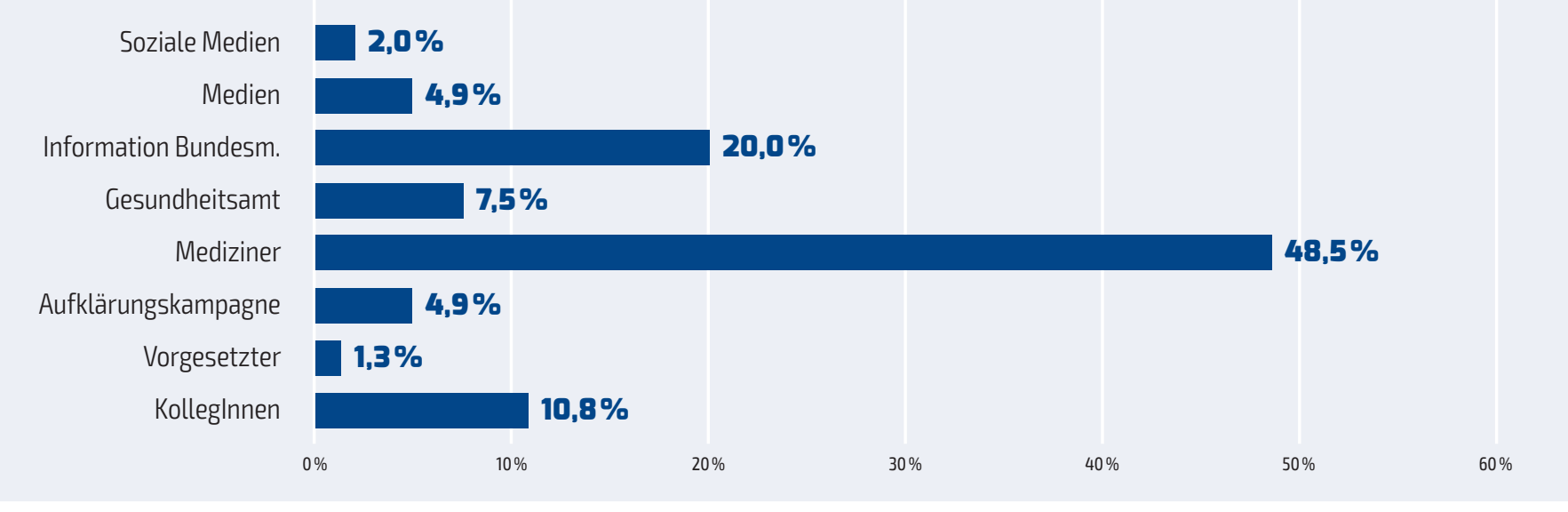


sche Untersuchung einmalig durchgeführt wird. Bei einer Querschnittuntersuchung werden Vorgehensweisen und Meinungen von Teilnehmern unterschiedlicher Altersstufen zu einem festen Untersuchungszeitpunkt miteinander verglichen. So entstehen gesellschaftliche Momentaufnahmen von derzeit gültigen Fakten, Meinungen oder Verhaltensweisen.

\section{Vertrauen in die Impfstrategie}

Das Ziel der Befragung war es, die persönlichen Motivationen für die Teilnahme an der Schutzimpfung der Mitarbeiter des KKW zu erheben, ebenso den Wissensstand der geimpften Personen in Bezug auf die neu entwickelte COVID-19-Schutzimpfung zu eruieren. Die Zielgruppe waren alle zu diesem Zeitpunkt geimpften Mitarbeiter des Klinikums Klagenfurt aus unterschiedlichen Berufsgruppen der Kategorie I. Die erfassten Daten wurden automatisch in entsprechende Datenbanken, Grafiken und Kreuztabellen umgewandelt. Die Fragebögen wurden nach der elektronischen Auswertung durch das Qualitätsmanagement des Hauses gespeichert und durch die Sachgebietsleitung Gesundheitswissenschaften ausgewertet. Die aktuelle Befragung unterliegt den Datenschutzrichtlinien der KABEG und wurde anonym durchgeführt.

Auf die Frage, ob die Mitarbeiter Vertrauen in die aktuelle Impfstrategie für Österreich haben, antworteten 80,5 Prozent der Befragten (649 MA) mit Ja; 2,7 Prozent der Befragten mit Nein und 16,8 Prozent der Befragten (138 MA) mit teilweise.

Auf die Frage: „Haben Sie die bisher allgemeinen empfohlenen Standardimpfungen des österreichischen Impfplans in Anspruch genommen?“, antworteten 90,6 Prozent (742 MA) der Befragten mit Ja. Diese Aussage lässt auf eine generell hohe Impfbereitschaft der Mitarbeiter im Gesundheitswesen schließen. Auf die Frage, ob die geimpften Mitarbeiter bei Reisen die dementsprechenden Impfempfehlungen in Anspruch nehmen, gaben 87,7 Prozent der Befragten (718 MA) an, diesen Empfehlungen nachzukommen.

Die Frage: „Welcher dieser Informationsquellen vertrauen Sie für eine Urteilsbildung für Impfungen am meisten?" wurde wie folgt beantwortet: 48,5 Prozent (397 MA) der Befragten gaben den Arzt ihres Vertrauens als wichtigste Informationsquelle für die Urteilsbildung an. Die Informationen seitens des Bundesministeriums für Soziales, Gesundheit, Pflege und Konsumentenschutz (BMSGPK) gaben 20 Prozent (164 MA) der Befragten als vertrauensvolle Informationsquelle für die Urteilsbildung an. 10,8 (88
MA) Prozent der Befragten geben die Kollegen in ihrem Arbeitsumfeld als für die Urteilsbildung wichtige Informationsquelle an. Der unmittelbare Vorgesetzte hatte mit nur 1,3 Prozent als Informationsquelle für die Urteilsbildung keinerlei Relevanz (siehe Abbildung 1, S. 48).

Auf die Frage, wie die Mitarbeiter auf die aktuelle COVID19-Schutzimpfung aufmerksam geworden sind, konnten folgende Bezugsquellen genannt werden: 24, 4 Prozent der Befragten gaben an, durch ihren unmittelbaren Vorgesetzten auf die COVID-19-Schutzimpfung aufmerksam geworden zu sein. Ebenfalls 24,4 Prozent wurden durch die Informationskampagne seitens des Bundesministeriums (BMSGPK) auf die Impfung aufmerksam. 19, 5 Prozent der befragten Mitarbeiter wurden über die Medien auf die COVID-19-Schutzimpfung aufmerksam und 11,11 Prozent über den Austausch mit Kollegen.

Die Frage, wie die Aufklärung über die COVID-19-Schutzimpfung und den verabreichten Impfstoff seitens des Arbeitgebers durch die geimpften Mitarbeiter bewertet wurde, führte zu folgendem Ergebnis: 32,8 Prozent der Befragten (269 MA) fühlten sich von ihrem Arbeitgeber sehr gut über die COVID-19-Schutzimpfung aufgeklärt, 40,0 Prozent (328 MA) gut und 26,3 Prozent unzureichend bis mangelhaft aufgeklärt.

\section{Schutz vor Krankheit und Freiheit zurückgewinnen}

Auf die Frage: „Sie haben sich für die neu entwickelte COVID19-Schutzimpfung entschieden. Was hat Sie für diese Entscheidung beeinflusst?" gaben die Befragten folgende, für sie wichtige Einflussfaktoren wieder (Mehrfachnennungen waren möglich): 51,8 Prozent der Befragten (424 MA) gaben an, sich durch die Informationen der nationalen Experten für den neu entwickelten COVID-19-Impfstoff entschieden zu haben. 37,2 Prozent gaben Informationen im Arbeitsbereich als Entscheidungsursache an und 33,2 Prozent der Befragten hatten sich im Rahmen ihrer Selbstrecherche für die neu entwickelte COVID-19-Schutzimpfung entschieden. 17,1 Prozent der Befragten machten ihre Entscheidung von den Informationen durch den Arzt ihres Vertrauens abhängig.

Auf die Frage nach den persönlichen Gründen für die COVID19-Schutzimpfung (Mehrfachnennungen waren möglich) gaben 60,7 Prozent der Befragten (497 MA) an, sie arbeiten in einem Krankenhaus und haben dadurch ein erhöhtes Risiko mit dem Virus in Kontakt zu kommen. Des Weiteren gaben sie an, durch

\section{ABB. 2: PERSÖNLICHE GRÜNDE FÜR DIE IMPFUNG}

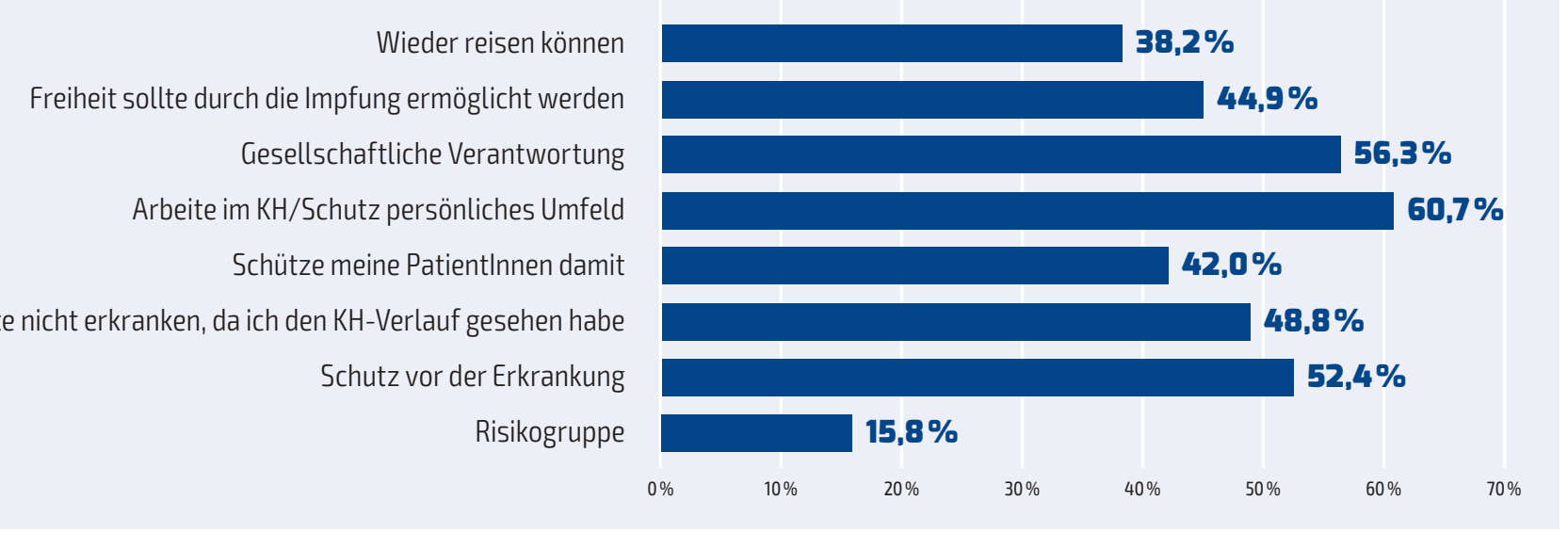


die Impfung ihr persönliches Umfeld schützen zu wollen. 56,3 Prozent (461 MA) der Befragten gaben an, ihre gesellschaftliche Verantwortung ernst zu nehmen (hohe Durchimpfungsrate notwendig). 52,4 Prozent (429 MA) gaben an, sie nutzen die Impfung, um sich vor der Krankheit zu schützen, des Weiteren gaben diese Personen an, generelle Impfbefürworter zu sein. 48 Prozent (393 MA) der Befragten haben in ihrem Handlungsfeld gesehen, wie diese Erkrankung verlaufen kann und möchten deshalb nicht daran erkranken. 44,9 Prozent (367 MA) der befragten Mitarbeiterinnen und Mitarbeiter möchten ein wenig Freiheit zurückbekommen und sehen die Impfung als Chance, diese wiederzuerlangen. 42 Prozent der Befragten gaben an, sie würden durch die eigene Impfbereitschaft auch die Patienten schützen (siehe Abbildung 2, S. 49).

Auf die Frage nach der Bewertung des Aufklärungs- und Dokumentationsbogens (inhaltliche Schwerpunkte, Verständlichkeit und Nachvollziehbarkeit der Fragen und der zu erhebenden Punkte...) des Bundesministeriums zur Schutzimpfung gaben die Mitarbeiter Folgendes an: 48,4 Prozent (396 MA) bewerteten den Aufklärungs- und Dokumentationsbogen als gut, 22,1 Prozent als sehr gut und 29,4 Prozent der befragten Mitarbeiter bewerteten diesen als befriedigend bis mangelhaft.

\section{Gute Aufklärung}

Die Phase 1 der COVID-19-Schutzimpfungen am Klinikum Klagenfurt am Wörthersee konnte mit einem sehr guten Ergebnis und einer hohen Zufriedenheit unter den geimpften Mitarbeitern begonnen werden. Die geimpften Mitarbeiter fühlen sich über den Ablauf der Impfstraße und des Impfprocedere ausreichend informiert.

Aussagen in der Befragung lassen auf eine generell hohe Impfbereitschaft der Mitarbeiter im Gesundheitswesen und ein hohes Verantwortungsgefühl ihrem persönlichen Umfeld und den Patienten gegenüber schließen. Auch der Wunsch der Mitarbeiter, mithilfe der COVID-19-Schutzimpfung ein Stück persönliche Freiheit wiederzuerlangen, wurde im Rahmen der persönlichen Gründe klar ersichtlich.

Abschließend kann bemerkt werden, wie wichtig eine gute Aufklärung, ausreichende Informationsmöglichkeiten für Betroffene und aufgrund der begrenzten Impfkapazitäten eine gut durchdachte Impfstrategie sind. In den nächsten Wochen werden die restlichen Mitarbeiter der Kategorie 1 und der weiteren Kategorien geimpft.

\section{Kurzinformation für Gesundheitspersonal: COVID-19-Impfstoffe auf mRNA Basis Stand: 15. Dezembe 2020: https://www.sozialministerium.at/Informationen-zum-Coronavirus/COVID-19-Impfung.html Zugriff: 02.02 .2021 \\ 2, 3 Bundesministerium Soziales, Gesundheit, Pflege und Konsumentenschutz: COVID-19-Impfungen: Empfehlungen des Nationalen Impfgremiums zur Priorisierung Version 2.1, Stand: 26.12 .2020 \\ 4 COVID-19-Impfung: Impfstrategie für Österreich - Umsetzung und Durchführung Version 1.0, Stand: 21. Dezember 2020}

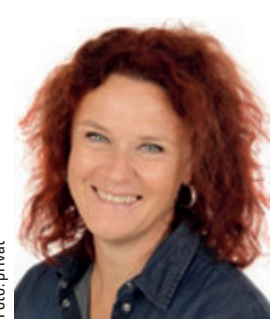

\section{MMAG. ${ }^{A}$ CHRISTINE LEBER-ANDERWALD}

Pflegedirektion/Sachgebietsleitung Angewandte Gesundheitswissenschaft/Health Science, Klinikum Klagenfurt am Wörthersee christine.leber@kabeg.at

\section{Lernaffekt}

$\mathrm{Zu}$ Mückstein sind schon vieler Kommentare. Ihr A. di Positas beschränkt sich vorerst darauf, seiner großen Sorge um die offensichtlich ins Bodenlose gesunkene Attraktivität des Arztberufes, im Speziellen des Allgemeinmediziners und noch genauer des Arztes in einer Primärversorgungseinheit Ausdruck zu verleihen.

Zum erhofften Lernen aus Krisen ernüchtere das Archiv unseres Schwestermediums QUALITAS (1/2010): Die Ereignisse rund um die von der WHO als Pandemie eingestufte „Neue Grippe“-von Gerd Antes als das bisher größte Desaster im Gesundheitssystem bezeichnet-können als Paradebeispiel herhalten, wie wichtig es ist, evidenzbasierte Medizin nicht nur in der Arzt-Patientenbeziehung, sondern auch in der Gesellschaft zu verankern. Das Rezept für das „Schweinegrippen-Missmanagement“: Man führt eine künstliche Krise herbei und erzeugt durch das Zelebrieren von Einzelfällen einen Medienhype, der zu einem Kommunikationschaos führt. Im Oktober 2009 meldete eine deutsche Illustrierte „Wir müssen mit 35.000 Toten rechnen."Aus Gesunden werden dann besorgte Gesunde. Das Rad beginnt sich schneller zu drehen und ist bald nicht mehr aufzuhalten. Entscheidungsträger geraten unter Druck. Viele Staaten konnten

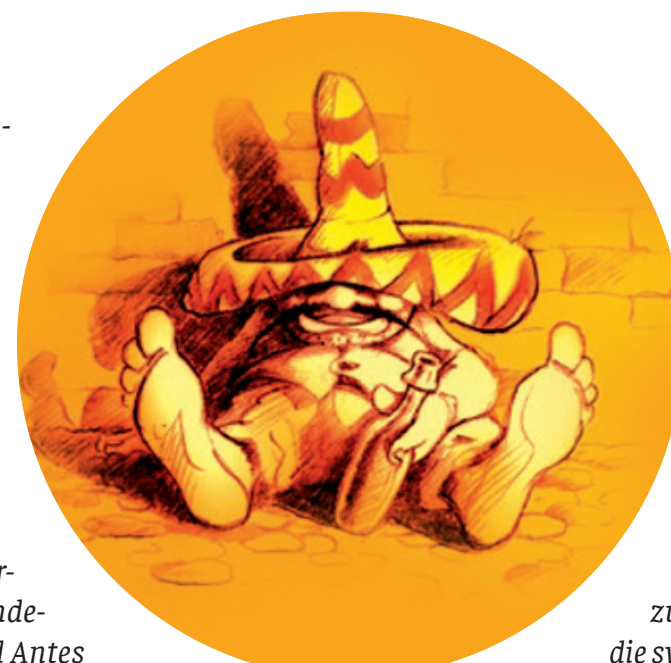

dazu bewegt werden, durch eine Bevorratung gesicherte Absatzmärkte für die Impfstoffe zu erzeugen. Wissenschaftler und Skeptiker wurden in Entscheidungsgremien mit Schweigepflicht berufen und so mundtot gemacht. „Das haben wir viel zu spät registriert“, stellt Claudia Wild fest. Die Frage „Haben wir das Richtige richtig getan?" wurde nicht gestellt. Thomas Pieber sieht im Umgang mit der Neuen Grippe ein gutes Lehrbeispiel dafür, wie schwer evidenzbasierte Medizin und Krisen zusammenpassen. Eine Krise ist akut, Zeit für die systematische Aufarbeitung von Daten - sofern sie denn überhaupt erhoben werden - bleibt nicht. Martin Sprenger geht noch einen Schritt weiter: „Krisen schränken das rationale Denken ein." [...] Die Diskutanten fassen [...] zusammen: Wir müssen lernen und Ärzten, Patienten, Gesundheitspolitikern und anderen Entscheidungsträgern beibringen, wie man Unsicherheiten kommuniziert. Wir müssen in Krisenzeiten auf eine Evidenzgenerierung hinarbeiten. Wir brauchen mehr Transparenz im System und eine schonungslose Offenlegung von Interessenkonflikten.

Mit ewig-neuem Handkuss

Ihr A. DI POSITAS 